\title{
CVD Flow Field Modeling Using the Quiet Direct Simulation (QDS) Method
}

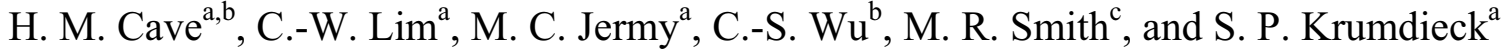 \\ ${ }^{a}$ Department of Mechanical Engineering, University of Canterbury, Private Bag 4800, \\ Christchurch 8140, New Zealand. \\ ${ }^{b}$ Department of Mechanical Engineering, National Chiao-Tung University, Hsinchu, \\ Taiwan. \\ ${ }^{\mathrm{c}}$ National Centre for High Performance Computing, Hsinchu, Taiwan.
}

In this paper, the Quiet Direct Simulation (QDS) method is used to model the unsteady jet development in a Pulsed Pressure Chemical Vapour Deposition (PP-CVD) reactor. QDS is a novel method of gas flow simulation which is able to compute true-direction fluxes of mass, momentum and energy in a computationally efficient and accurate manner. The scheme is ideal for the simulation of novel CVD processes like PP-CVD which include highly unsteady flow structures which has previously proved extremely difficult to simulate. Here, the axisymmetric QDS solver is outlined and the injection phase of a PP-CVD reactor is simulated.

\section{Introduction}

CVD flow field modelling is typically carried out using commercial computational fluid dynamics software (CFD) for higher pressure systems. Contemporary finite volume CFD codes divide the computational domain into a grid of finite volumes and fluxes of mass, momentum and energy are calculated through the interfaces between these volumes using discretized Navier-Stokes equations. In this technique, poor alignment of the grid with the flow field results in errors since fluxes can only occur between volumes which share an interface. Thus, CFD requires careful grid design to ensure accurate results, convergence and stability.

Alternatively, CVD flow fields at lower pressures can be modelled using the Direct Simulation Monte Carlo (DSMC) technique (1). In DSMC, the flow is simulated by tracking the movement of a large number of simulated particles through the flow field. Particles are allowed to move between any two points on the grid and, as such, fluxes of properties in DSMC are true-direction and grid independent. After the convection of the particles over a time step, the particles are indexed to the grid and nearby particles selected to suffer scattering collisions. These collisions are generated on a probabilistic basis requiring the use of random numbers for collision partner selection and to determine the likelihood of the collision actually occurring. As such, DSMC is subject to statistical scatter and requires averaging over a large number of time steps to reduce the scatter in the sampled macroscopic properties resulting in considerable computational expense.

In present work a novel kinetic-based technique called the Quiet Direct Simulation (QDS) method is presented. The QDS method was originally developed and implemented to first order accuracy by Albright et al. (who originally called the method QDSMC, or Quiet Direct Simulation Monte Carlo) as a method for modelling plasmas 
(2) and simple Eulerian flows (3). QDSMC was reformulated as a conservative finite volume scheme and implemented to second order accuracy by Smith et al. and renamed as QDS due to the lack of stochastic processes (4). Cave et al. then carried out a nontrivial extension of the second-order QDS scheme to an axisymmetric solver (5). In QDS, true-direction fluxes of properties between computational cells are determined from an approximate Maxwell-Boltzmann distribution where the mass carried by the fluxes and their velocities are represented by the weights and abscissas of a Gauss-Hermite quadrature. The QDS algorithm requires no evaluation of complex functions and does not suffer from the statistical scatter inherent in the DSMC solvers, while its truedirection fluxes ensure accuracy on grids which are not aligned with the flow field. In addition QDS has a large dynamic range, is easily extendable to multi-dimensions and multi-species, is computationally inexpensive and is easily implemented on parallel computers.

In this paper, an axisymmetric second-order QDS scheme is introduced to simulate the flow field in a Pulsed Pressure CVD (PP-CVD) reactor. The PP-CVD process is characterized by a highly unsteady flow field which results in highly uniform thin films of a variety of materials with high precursor conversion efficiencies $(6,7)$. The operating principle of pulsed-pressure CVD is a rapid injection of the precursor into a continuously evacuated low pressure reactor volume in timed pulses, followed by a pump-down evacuation cycle (8). This process produces a reactor pressure pattern shown in Figure 1. Previous attempts to model PP-CVD with DSMC solvers have proved extremely challenging and computationally expensive (9); however, QDS scheme allows the rapid simulation of problems on standard computer equipment.

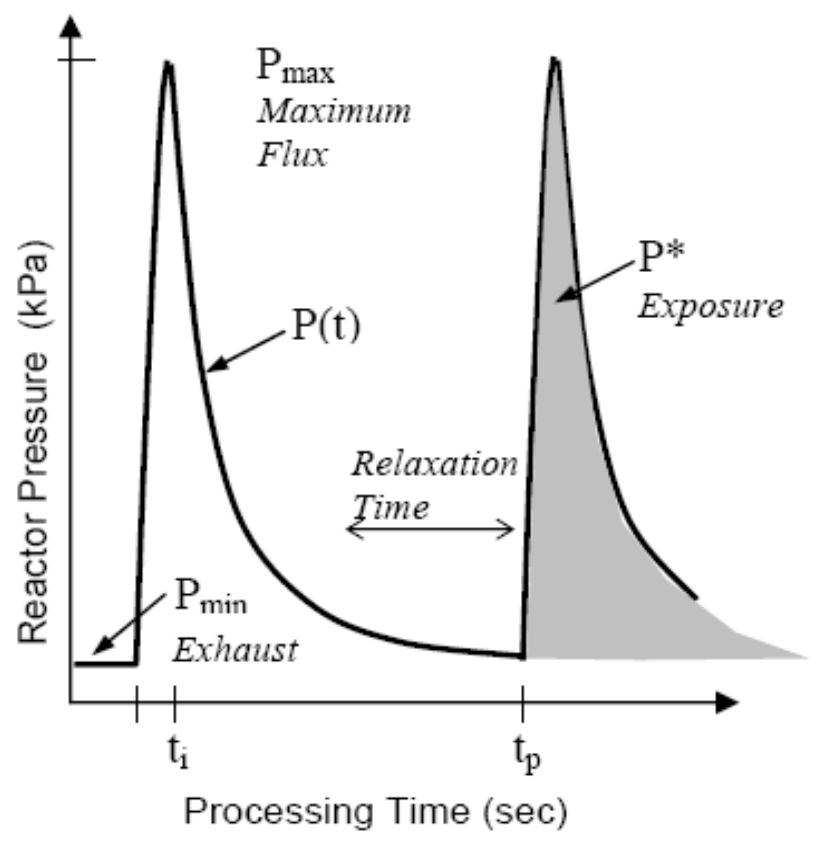

Figure 1. PP-CVD reactor pressure during one pulse cycle 


\section{Method}

In QDS, the flow is assumed to have a Maxwell-Boltzmann equilibrium velocity distribution:

$$
p(v)=\frac{1}{\sqrt{2 \pi} \sigma} \exp \left(\frac{-(v-u)^{2}}{2 \sigma^{2}}\right)
$$

where $u$ is the bulk velocity and the velocity variance, $\sigma=\sqrt{R T}$.

By using a Gaussian quadrature approximation, the integral of equation [1] over its limits can be approximated by:

$$
\int_{-\infty}^{\infty} \frac{e^{-v^{2} / 2 \sigma^{2}}}{\sqrt{2 \pi \sigma}} f(x) d x \approx \sum_{j=1}^{N} w_{j} f\left(q_{j}\right)
$$

where $w_{j}$ and $q_{j}$ are the weights and abscissas of the Gaussian quadrature (also known as the Gauss-Hermite parameters). Thus, in QDS the Maxwell-Boltzmann equilibrium distribution of velocities can be approximated with a small number (usually three or four) of representative fluxes in each coordinate direction. The flux velocities are determined by the abscissas of the quadrature, and the fraction of cell mass carried in each flux is determined by the associated weights.

In the current QDS algorithm, the $2 N$ flux scheme employed by Smith et al. (10) is used to calculate these two dimensional true direction fluxes of mass, momentum and energy between cells. A second order scheme is employed, in which the gradients of cell velocity in the $x$-coordinate direction $\left(d u_{x} / d x\right)$, can be used to update the flux velocity:

$$
v_{x j}=u_{x}+\frac{d u_{x}}{d x} \Delta x_{L}+\sqrt{2 \sigma_{v}^{2}} q_{j}
$$

where $\Delta x_{L}$ represents the location in the cell from where the flow properties are taken, corresponding to the displacement of the centre of mass of the flux which moves into the destination cell.

The energy carried by each flux is given by:

$$
\varepsilon_{x j}=\frac{(\xi-\Omega)\left(\sigma_{v}+\frac{d \sigma_{v}}{d x} \Delta x_{L}\right)^{2}}{2 \Omega}
$$

where $\xi$ is the total number of degrees of freedom $(\xi=2(\gamma-1)-1)$ and $\Omega$ is the number of simulated translation degrees of freedom. 
The mass carried by axial components of fluxes is given by:

$$
m_{x j}=\frac{\left(\rho+\frac{d \rho}{d x} \Delta x_{L}\right) \Delta x w_{j}}{\sqrt{\pi}}
$$

The total mass carried by radial fluxes is determined using the method outlined in Cave et al. (5). Since fluxes moving in a radial direction in an axisymmetric flow undergo a change of volume, the derivation of these fluxes is non-trivial and interested readers are asked to read reference (5). As an example, a flux moving away from the axis has mass:

$$
m_{r j}=\frac{\alpha \Delta x\left[\frac{B}{3}\left(r_{R}^{3}-r_{1}^{3}\right)+\frac{C}{2}\left(r_{R}^{2}-r_{1}^{2}\right)\right] w_{y j}}{\sqrt{\pi}}
$$

where the density gradient across the cell $B=d \rho / d r, C=\rho_{c m}-B r_{c m}, r_{R}$ is the upper boundary of the cell, $r_{c m}$ is the centre of mass of the source cell, $\alpha$ is the axisymmetric angle (usually $\pi$ ) and $r_{l}=r_{R}-v_{r j} \Delta t$.

An additional momentum correction is also required to account for the variation in the force due to static pressure across the top and bottom interfaces of the axisymmetric cell. An amount of momentum is added to each cell equal to:

$$
\Delta M=P \alpha \Delta r \Delta x \Delta t
$$

where $P(=\rho R T)$ is the pressure in the cell, $\Delta r$ is the radial size of the cell and $\Delta t$ is the simulation time step.

Once the associated flux properties of each cell are determined, fluxes of mass, momentum and energy are exchanged between cells. The amount of mass which advances to an adjacent cell can be determined by multiplying equation [5] by $v_{x j} \Delta t / \Delta x$, hence the exchanged mass, momentum and energy (for the axial direction) are:

$$
\begin{gathered}
m_{f l u x, x}=\frac{v_{x j} \Delta t}{\Delta x} m_{x j} \\
p_{f l u x, x}=\frac{v_{x j} \Delta t}{\Delta x} m_{x j} v_{x j} \\
E_{f l u x, x}=\frac{v_{x j} \Delta t}{\Delta x} m_{x j}\left[\frac{1}{2} v_{x j}^{2}+\varepsilon_{x j}\right]
\end{gathered}
$$

To reduce the numerical diffusivity of the scheme, the gradients used in equations [3]-[6] are generated using a monotonized central differencing flux limiter (11) and the simulation time step $\Delta t$ is set dynamically to maintain the maximum kinetic Courant- 
Friedrichs-Levy (CFL) number in the domain below a desired value (usually $\leq 1$ ). It is important to note that this CFL restriction is to maintain physical realism and is not related to the numerical stability of the scheme.

\section{Results and discussion}

The QDS technique has been extensively verified and validated against CFD and direct simulation (DS) solvers for two-dimensional and axisymmetric steady and unsteady flows; and its implementation performance have been reported in detail by Smith et al. (4) and Cave et al. (5). The method has been shown to be considerably faster than both CFD and DS solvers, while maintaining computational stability and accuracy compared to benchmarking cases.

Here, the QDS method is used to simulate a basic Pulsed Pressure CVD (PP-CVD) reactor. PP-CVD is a novel CVD technique with improved performance over traditional CVD methods in several important areas, including film quality and substrate conformity (7), and also demonstrates very high precursor conversion efficiency (12) and high film growth rates (13). In PP-CVD, the timed injection of a controlled volume of precursor into a continuously evacuated reactor volume results a highly unsteady flow field through the reactor volume.

The PP-CVD flow field has been investigated experimentally by Krumdieck et al. (14) and modelled using unsteady DSMC simulations by Cave (9). Unfortunately, the highly unsteady nature of the flow coupled with the large density gradients and rarefaction makes experimental flow visualization very challenging, and DSMC simulations are extremely computationally expensive. Conventional CFD solvers are also computationally expensive and obtaining converged solutions is often difficult. For this reason, QDS has been identified as a potential method for investigating the PP-CVD flow field and for reactor development simulations.

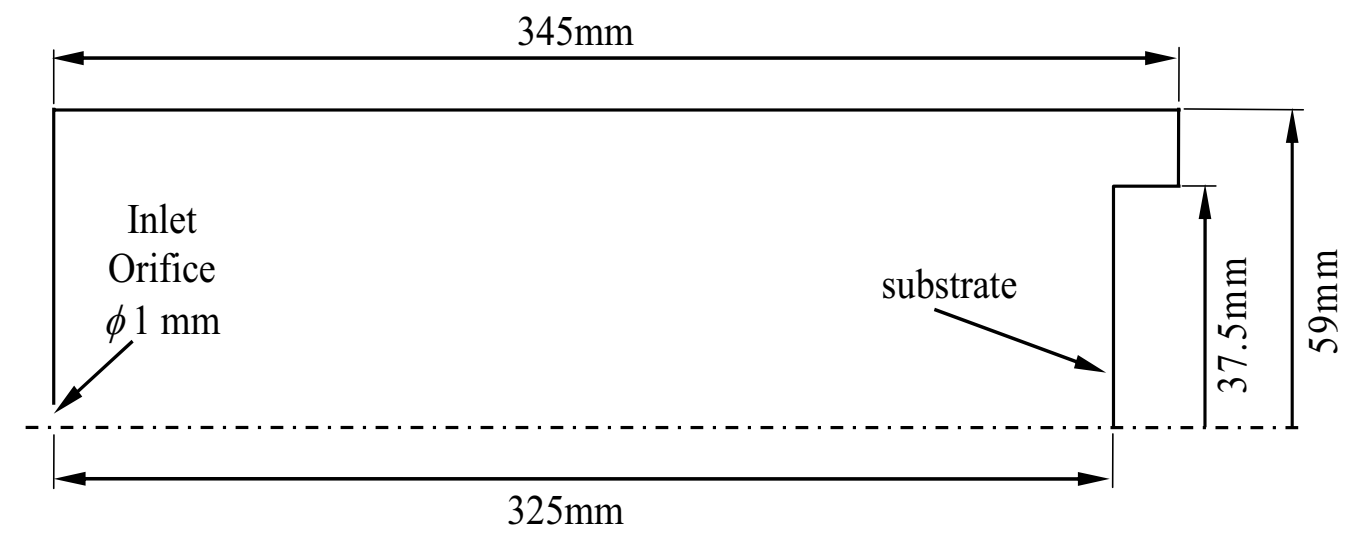

Figure 2. Schematic of Pulsed Pressure CVD reactor geometry.

The geometry of the simulation is shown in Figure 2. Ideal helium is used as the simulation gas, having a ratio of specific heats of 1.67 and a gas constant of $R=2077$ $\mathrm{J} /(\mathrm{kg} \cdot \mathrm{K})$. The gas, at a supply pressure of $400 \mathrm{kPa}$ and a temperature of $293 \mathrm{~K}$, is injected into the reactor though an orifice resulting in a choked inlet flow condition. The reactor 
volume is initially filled with stationary gas at a pressure of $1 \mathrm{kPa}$ and a temperature of 293K. The second-order axisymmetric QDS solver utilized a variable time step scheme such that CFL $<0.5$ and used four QDS fluxes in each coordinate direction in a $2 N$ flux scheme. The simulations were carried out using 312,744 square cells. The simulations required approximately 24 hours to simulate $10 \mathrm{~ms}$ of flow time on a $3.00 \mathrm{GHz}$ Intel Core 2 Duo CPU with 4GB of RAM. This was sufficient to bring the flow to a quasi-steady state during the injection phase.

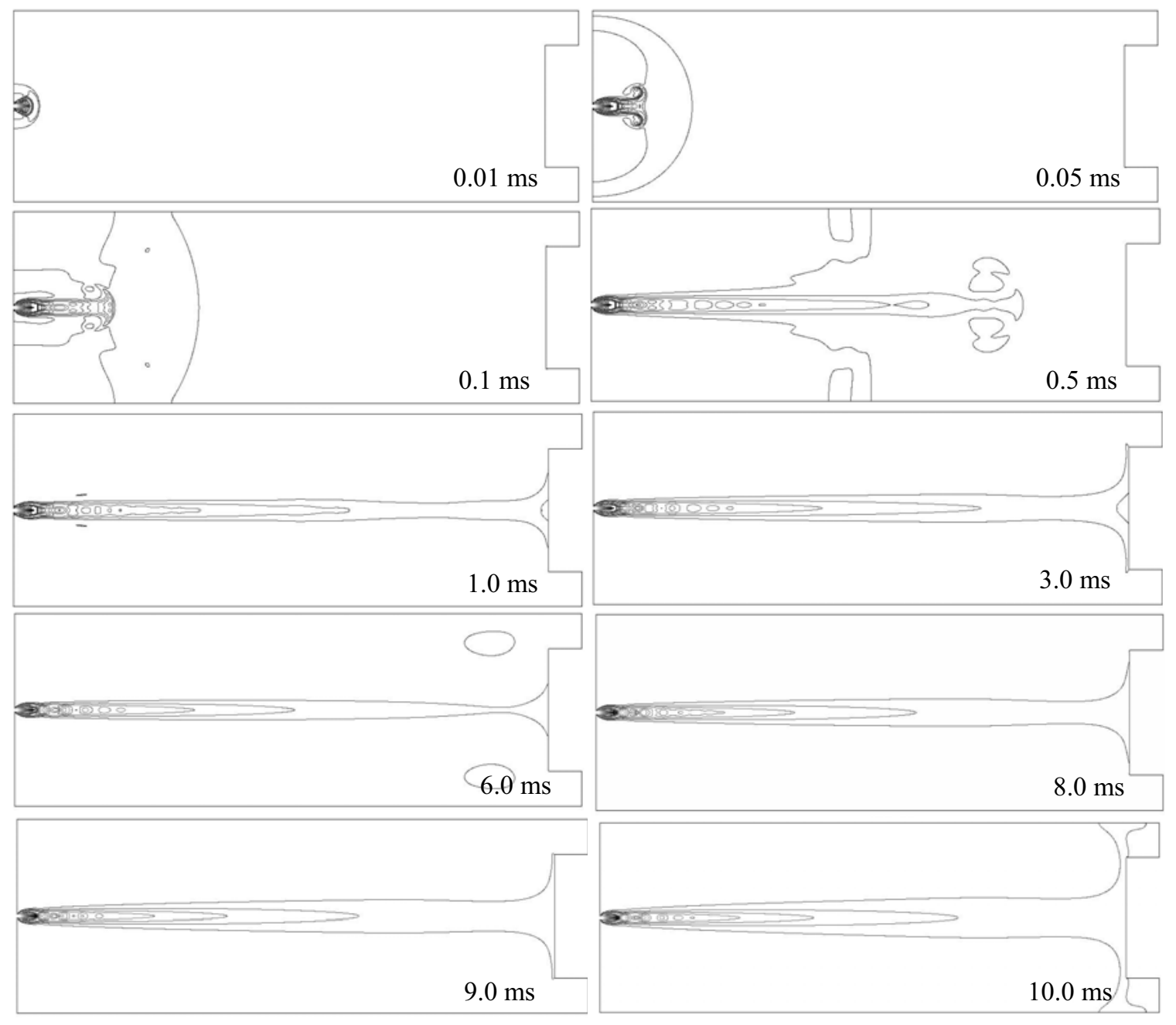

Figure 3. Contours of $\log$ (density) for the unsteady development of an under-expanded jet in a PP-CVD reactor.

Figure 3 shows the unsteady flow development in the reactor as the under-expanded jet develops during the injection phase. These results capture the shock structure and flow development with far higher resolution and much lower computational expense than the DSMC simulations in reference (9), although under the assumption that the flow is in thermal equilibrium. As such, axisymmetric QDS represents a viable method for the rapid approximate simulation of PP-CVD reactors while these are being developed for specific applications.

For the assumption of thermal equilibrium to be correct, the number of collisions per particle per time step in a cell should be greater than 4 . This number of collisions is 
sufficient to bring the flow approximately into equilibrium (15). In the current simulation, the number of collisions per particle per time step at the inlet is about 200 and 1.65 in the quiescent regions, which is acceptable as the gas is near stationary here, allowing equilibrium to re-establish before the macroscopic state changes significantly. The number of collisions per particle per time step is also less than unity in the Mach discs and some non-quiescent, low pressure regions. To further investigate the validity of thermal equilibrium assumption, the gradient-length local (GLL) Knudsen number based on density proposed by Boyd and Chen (16) was calculated at various time steps. As studied by Boyd and Chen, continuum breakdown occurs when GLL Knudsen number is greater than 0.05. The current simulation results show that the GLL Knudsen number of the flow is generally less than 0.01 except in limited regions in the shocks where the GLL Knudsen number is about 0.1 , however this region is unlikely to have a significant effect on the flow field. Therefore, it can be said that continuum breakdown generally did not occur in the flow field simulated. This indicates that although the number of collisions was low in some regions of the flow, the local density gradient within the computational cell was not high enough to transport the particles downstream before thermal equilibrium condition was established. In addition, future work addressing a hybrid QDS-DSMC method has also been identified as a method to deal with regions of the flow which cannot be guaranteed to meet the equilibrium condition.

In order to enable the evaluation of design parameters of the PP-CVD reactor, mass flux of the precursor onto the substrate (MFLOS) and the accumulated mass of precursor onto the substrate (AMOS) are also computed. Figure 4 shows MFLOS and AMOS per area for the $20 \mathrm{~ms}$ injection phase simulation of the PP-CVD reactor flow field shown in Figure 3. Since the simulation was conducted for only $10 \mathrm{~ms}$ of the injection phase, both MFLOS and AMOS do not demonstrate uniformity, however these are likely to be important metrics for full reactor simulations.

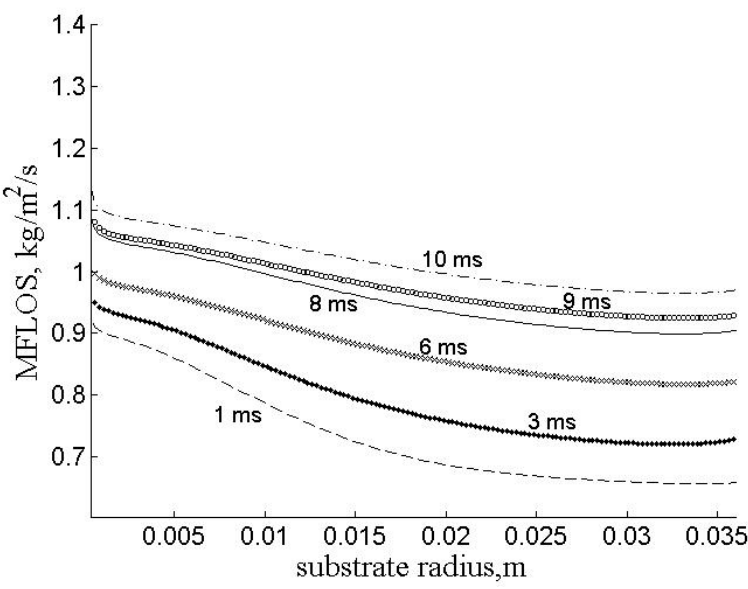

a

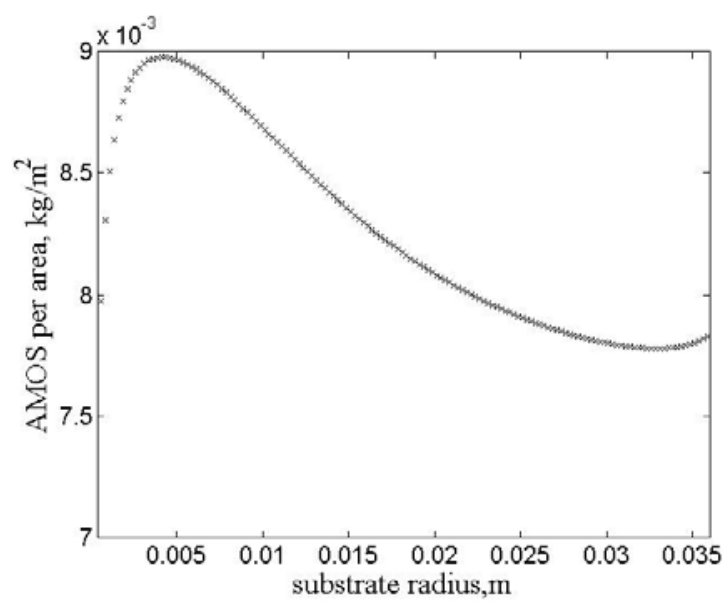

b

Figure 4. The plots of mass flux of the precursor onto the substrate, MFLOS (a) and the accumulated mass of precursor onto the substrate, AMOS (b) for the unsteady flow development in a PP-CVD reactor. 


\section{Conclusion}

In this paper an axisymmetric, second-order scheme Quiet Direct Simulation (QDS) method was introduced for the flow field simulation of a PP-CVD reactor. The rapid simulation time, accurate results and high quality flow visualization show QDS to be a viable and promising design tool for CVD systems such as PP-CVD which involves unsteady flow development throughout the deposition process.

\section{Acknowledgments}

This work was supported by the New Zealand Foundation for Research, Science and Technology (FRST) International Investment Opportunity Fund (IIOF) (grant no. UOC40210) and the National Science Council of Taiwan (grant no. 96-2628-E-009-136MY3).

\section{References}

1. G. A. Bird, Molecular Gas Dynamics and the Direct Simulation of Gas Flows, Clarendon Press, Oxford (1994).

2. B. J. Albright, W. Daughton, D. S. Lemons, D. Winske and M. E. Jones, Phys. Plasma, 9(5), 1898 (2002).

3. B. J. Albright, D. S. Lemons, M. E. Jones and D. Winske, Physical Review E, 65, 1 (2002).

4. M. R. Smith, H. M. Cave, Y. -S. Chen, M. C. Jermy and J. -S. Wu, Journal of Computational Physics, 228, 2213 (2009).

5. H. M. Cave, M. R. Smith, J. -S. Wu, M. C. Jermy, S. P. Krumdieck, C. -W. Lim and K. -C. Tseng, Journal of Computational Physics, (in review 2009).

6. S. P. Krumdieck and R. Raj, Journal of the American Ceramic Society, 82(6), 1605 (1999).

7. S. P. Krumdieck, A. Kristinsdottir, L. Ramirez, M. Lebedev and N. Long, Surface \& Coatings Technology, 201(22-23), 8908 (2007).

8. S. P. Krumdieck, S. I. Baluti, L. Marcus and A. Peled, in Journal of the Electrochemical Society, p. 120, Proceedings of CVD-XVI/EUROCVD-15, Bochum, Germany, (2005).

9. H. M. Cave, Development of Modelling Techniques for Pulsed Pressure Chemical Vapour Deposition (PP-CVD), Ph.D. Thesis, University of Canterbury (2008).

10. M. R. Smith, M. N. Macrossan and M. M. Abdel-jawad, J. Comput. Phys., 227(8), 4142 (2008).

11. B. van Leer, J. Comput. Phys., 23, 263 (1977).

12. H. M. Cave, S. P. Krumdieck and M. C. Jermy, Chem. Eng. J., 135, 120 (2008).

13. S. P. Krumdieck and R. Raj, Journal of the American Ceramic Society, 82(6), 1605 (1999).

14. S. P. Krumdieck, J. -Y. Lee and H. Raatz, J. Electrochem. Soc., 8, 179 (2003).

15. A. Sharma and L. N. Long, Journal of Computational Physics, 200, 211 (2004).

16. I. D. Boyd and G. Chen, Phys. Fluids, 7(1), 210 (1995). 